\title{
Analysis of dynamic model based on pedestrian's abnormal posture
}

\author{
Rongyong Zhao, Ping Jia*, Chuanfeng Han, Yan Wang, Cuiling Li and Zhishu Zhang \\ School of Electronic and Information Engineering, Tongji University, Shanghai, China
}

\begin{abstract}
It is significant to detect abnormal postures of pedestrians in the crowd to crowd stability control. This study locates the joint points of pedestrians based on the pose estimation algorithm OpenPose. After the analysis of 18 nodes and six body parts, the sudden value of node acceleration is obtained, which is compared with the acceleration of the pedestrian's centre of mass. When there is at least one difference in the direction or acceleration value of the two, it means that the pedestrian has abnormal behaviour. Furthermore, this study analyses the result of comparing the change of $z$-coordinate value in pedestrian movement with $20 \%$ of pedestrian height. These two judgment methods together constitute the dynamic criterion of pedestrian abnormal posture, and judge whether the pedestrian has abnormal behaviour. Compared with the previous dynamic analysis of pedestrian abnormal posture, the accuracy of abnormal posture judgment is improved. This provides a theoretical basis for crowd stability analysis.
\end{abstract}

Keywords: Abnormal behaviour, OpenPose, Crowd stability analysis.

\section{Introduction}

Human body posture research began in the 1970s[1], and current application areas include security systems, human-computer interaction, sports assistance, clinical correction, and virtual reality. Gesture recognition is a process from low-level processing to high-level analysis from shallow to deep. Low-level processing is the basis of high-level analysis, including detection and tracking, trajectory analysis, and gesture recognition. The process of high-level analysis includes human feature extraction, posture modelling, scene relationships[2-4], etc. Gesture recognition is an important part of crowd risk identification. Public places are generally equipped with network video surveillance systems. The rapid development of computer vision technology also provides support for online gesture recognition.

At present, there are frequent stampede incidents in public places, and there are more and more disasters caused by abnormal pedestrian behaviours, such as pedestrians falling, pedestrian speed sudden changes and other abnormal behaviours. Pedestrian abnormal

*Corresponding author:2033108@tongji.edu.cn 
posture recognition is the focus of current human posture research. The analysis of the dynamic model of pedestrian abnormal posture in public places is particularly important. Gesture recognition can effectively detect the precursors of abnormal crowd disturbances. The use of human posture recognition technology to analyse the dynamic model of pedestrian abnormal posture is of great significance to the analysis of crowd stability in public places.

\section{Feature extraction of human posture}

Human posture recognition methods are mainly divided into vision-based human posture recognition and sensor-based human posture recognition. This study uses vision-based recognition of abnormal pedestrian gestures. Abnormal behaviour detection and gesture recognition of human motion are both in the field of human motion recognition. Human body motion recognition refers to the use of certain methods to detect and track the human body's motion, obtain the human body's motion parameters. Then human body motion recognition reconstructs the human body's structure and posture from it, and finally achieves the understanding of the human body's motion and apply it. In a broad sense, the research object of human movement recognition can be small-scale local movements represented by the face, lips, gestures, gait or the body movements of the whole body including limbs[5]. The pose estimation process mainly includes: image data acquisition, body boundary segmentation, body pose recognition and data classification[6]. Human body boundary segmentation is the basic part of gesture recognition, which uses key points of human bones to identify and output human skeleton information.

\subsection{Pedestrian abnormal behavior detection based on machine vision}

Deep learning methods use convolutional neural networks to learn features to estimate human pose. The purpose of human motion detection is to extract the changing area from the background image from the sequence image. It involves the segmentation of the image and the discrimination of the human body area. Image segmentation is a low-level feature extraction, which mainly uses the geometric information, colour information and motion information of the human body, the shape, contour, and the shape and relative position of each part; and the colour information refers to the skin and clothes colour of the moving target. And motion information refers to the motion correlation between the various parts of the moving target body and the overall motion trajectory. The geometric information of the human body includes the extraction and fusion of the pedestrian feature of the moving target. It belongs to the middle-level machine vision, including the silhouette of the human body, features, global features, and local features, etc. The local feature skeleton joint point information improves the recognition accuracy with a smaller granularity $[7,8]$.

\subsection{OpenPose human pose estimation algorithm}

This study uses OpenPose software to obtain human joint point data. The typical bottom-up two-dimensional keypoint detection algorithm at this stage is the OpenPose[9] human pose estimation algorithm. The key technologies of this framework are joint confidence map (PCM) and joint affinity field. OpenPose uses VGG and the training network as the skeleton. One branch predicts confidence map and the other branch predicts correlation vector field. First, feature extraction is performed on the original image, and then the two features are input to the convolutional neural network of the two branches. The coordinates of the joint points of the human body are returned from the image or video. Figure 1 shows 
the image rendered by OpenPose. Then, this study use BM (Bipartite Matching) to obtain the positional relationship between the parts, and finally integrates it into a joint point connection diagram. The image processed by OpenPose is shown in Figure 2.

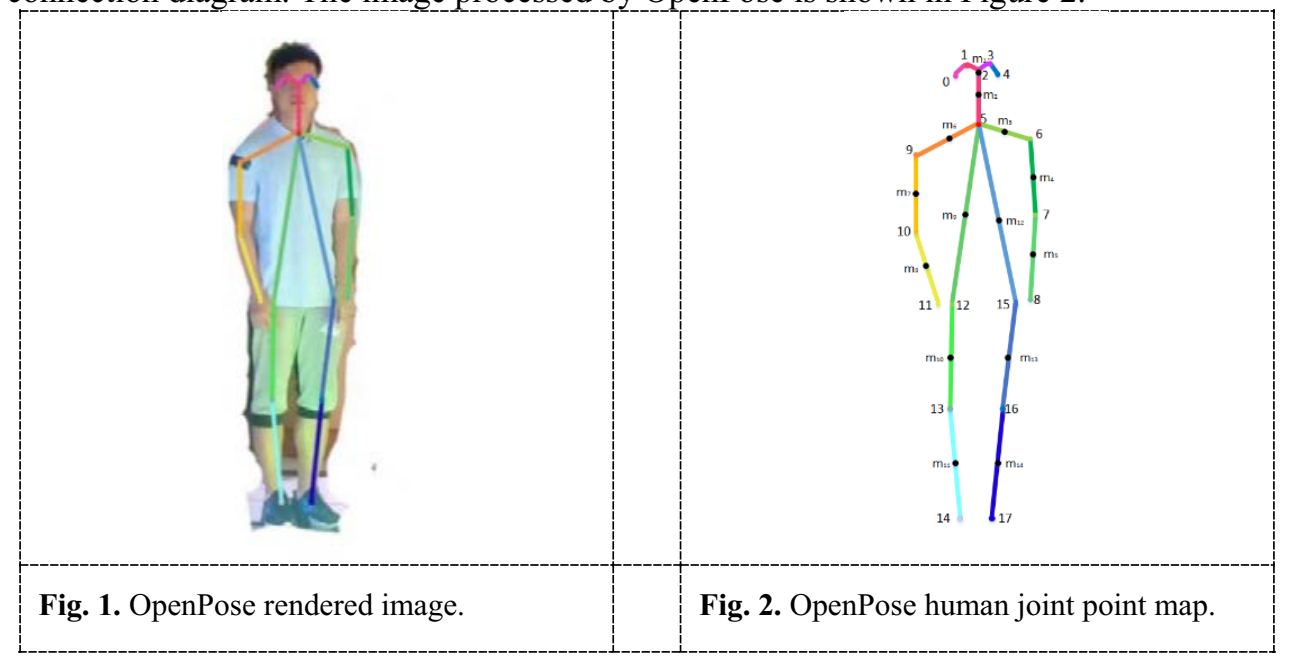

where $m_{1}$ to $m_{14}$ represent the centroids of each part. According to the specific positions, these joint points are divided into 6 subsets: $\left\{\right.$ head $B_{1}=\{0,1,2,3,4\}$, trunk $B_{2}=\{5,6,9,12,15\}$, right arm $B_{3}=\{10,11\}$, left arm $B_{4}=\{7,8\}$, right leg $B_{5}=\{13,14\}$, left leg $\left.B_{6}=\{16,17\}\right\}$.

\section{Dynamic analysis of pedestrian's abnormal posture}

Pedestrian abnormal postures include pedestrian falls, pedestrian speed changes, and pedestrian reverse behaviours. This study takes a pedestrian fall as an example, uses the OpenPose attitude estimation algorithm to obtain the joint data of the pedestrian's abnormal posture, locates the pedestrian's joint point and analyses the pedestrian's abnormal posture. The OpenPose human joint point map of the pedestrian's abnormal posture and the image rendered by OpenPose are shown in Figure 3 to 5.

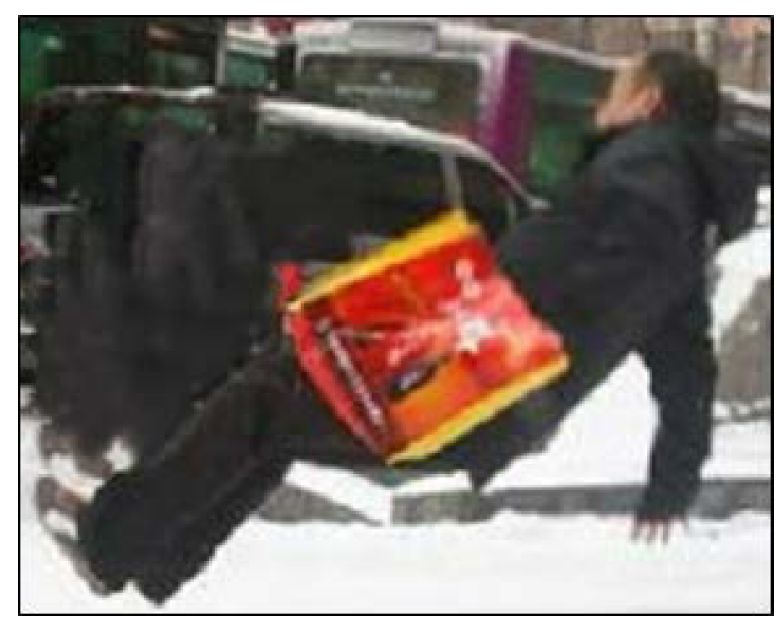

Fig. 3. Pedestrian abnormal posture image. 


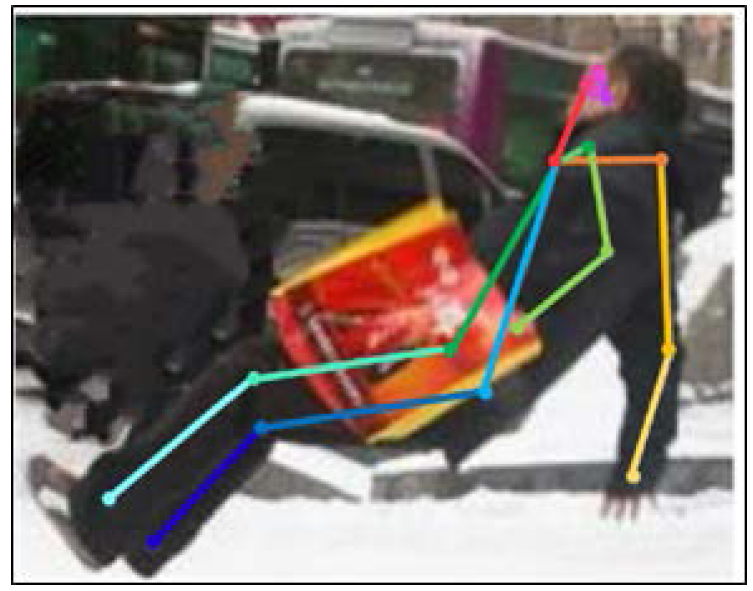

Fig. 4. OpenPose rendered image.

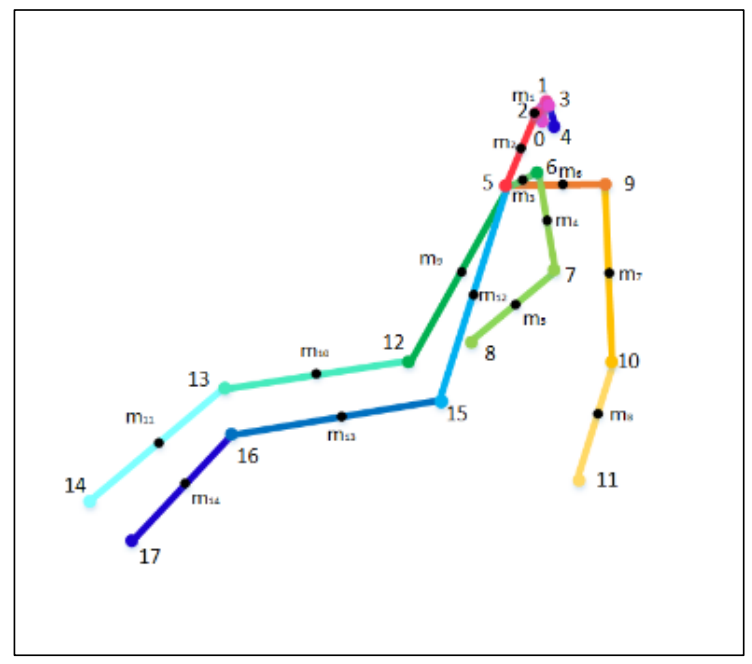

Fig. 5. OpenPose human joint point map.

For sports posture, we always involve the rigid displacement and rotation of the human skeleton. As we continue to change the local state of motion, the human skeleton will also produce changes in acceleration, motion speed and direction. The acceleration, movement speed and direction can effectively characterize the movement posture of our human body. Current research on abnormal pedestrian posture nodes usually converts pedestrian nodes into pedestrian mass points, calculates the position of the individual pedestrian's centre of mass, and calculates the individual's movement speed. The formulas for the centre of mass is shown in (1)-(5).

$$
\begin{aligned}
& x_{c}=\frac{\sum x_{i} m_{i}}{\sum x_{i}} \\
& y_{c}=\frac{\sum y_{i} m_{i}}{\sum m_{i}}
\end{aligned}
$$




$$
\begin{aligned}
z_{c} & =\frac{\sum z_{i} m_{i}}{\sum m_{i}} \\
r_{c} & =\frac{\sum r_{i} m_{i}}{\sum m_{i}} \\
\vec{v}_{c} & =\frac{d \overrightarrow{r_{c}}}{d t}
\end{aligned}
$$

where $r_{c}$ represents the vector diameter of the centre of mass, $x_{c}, y_{c}$ and $z_{c}$ respectively represent the $\mathrm{x}, \mathrm{y}$ and $\mathrm{z}$ coordinate values of the centre of mass, and $\boldsymbol{V}_{c}$ represents the speed of the centre of mass.

When a pedestrian moves, the coordinate values of the centre of mass in the $\mathrm{x}$ and $\mathrm{y}$ directions change all the time. When pedestrians exhibit abnormal behaviours, such as falling and other behaviours, a wide range of changes in the $\mathrm{z}$ direction may occur. According to experimental data, when the amount of change in the $\mathrm{z}$ direction exceeds $20 \%$ of the pedestrian's height, the pedestrian is considered to have an abnormal posture. The judgment method is shown in inequality (6) and $\mathrm{h}$ represents the height of the pedestrian.

$$
\Delta_{z} \geq 0.2 h
$$

When pedestrians fall, run fast and other events, the instantaneous speed of the centre of mass will change drastically or the direction of the speed will suddenly change, which means that the pedestrian's centre of mass has a sudden change. Regarding pedestrians as a mass point, the use of the speed and the position of the centre of mass has defects in judging the abnormal posture of the pedestrian, and it is difficult to identify the abnormal posture of the pedestrian. This study combines machine vision to collect image information, uses OpenPose to obtain human joint point maps, and analyses the acceleration, velocity, and direction of 18 nodes. In this study, after the analysis of dynamics and human joint points, the dynamics judgment equation of abnormal posture is constructed, as shown in equation (7).

$$
\overrightarrow{a_{c}}=\frac{\sum_{i=1}^{14} m_{i} \frac{d \overrightarrow{v_{i}}}{d t}}{\sum m_{i}}
$$

where $a_{c}$ represents the composite acceleration of the fourteen body parts, $v_{i}$ represents the speed of each body part and $m_{i}$ represents the mass of the body part corresponding to the node set.

When there is at least one difference between the direction and magnitude of $\overrightarrow{a_{c}}$ and the direction and magnitude of the acceleration of the centre of mass, it indicates that the pedestrian has the possibility of abnormal behaviour. Furthermore, combined with the result of comparing the change of z-coordinate value during pedestrian movement with $20 \%$ of the pedestrian's height, the dynamic criterion for the pedestrian's abnormal posture is formed together. 


\section{Summary}

In this study, the change in the pedestrian's centre of mass in the $\mathrm{z}$ direction is compared with $20 \%$ of the pedestrian's height to determine whether the pedestrian has abnormal behaviour. Furthermore, this paper analyses 18 nodes and 14 body parts, and obtains the sudden change value of node acceleration, which is compared with the acceleration of pedestrian's centre of mass. When there is at least one difference in the direction or acceleration value of the two, it means that the pedestrian has abnormal behaviour. Compared with the previous dynamic analysis of pedestrian abnormal posture, the accuracy of abnormal posture judgment is improved. This provides a theoretical basis for population stability analysis.

This research is financially supported by the National Natural Science Foundation of China (No.72074170).

\section{References}

1. I. Almeida, C. Jung. Crowd flow estimation from calibrated cameras[J]. Machine Vision and Applications, 2020, 32(1):312-320.

2. Z. Cao, T. Simon, S. Wei and Y. Sheikh. Realtime Multi-person 2D Pose Estimation Using Part Affinity Fields[C], 2017 IEEE Conference on Computer Vision and Pattern Recognition (CVPR), Honolulu, HI, USA, 2017, 1302-1310.

3. C. Patruno, R. Marani, G. Cicirelli, et al. People re-identification using skeleton standard posture and color descriptors from RGB-D data[J]. PATTERN RECOGNITION, 2019, 89:77-90.

4. T. V. Wouwe, L. H. Ting, F. D. Groote. Interactions between initial posture and tasklevel goal explain experimental variability in postural responses to perturbations of standing balance[J]. Journal of Neurophysiology, 2021, 125(2):586-598.

5. Yingzhuo Yu. Detection of Abnormal Behavior and Posture Recognition Based on Characteristics of Human Motion[D]. Xidian University,2011.

6. Y. Q. Kuang, M. Guo, Y. L. Peng, et al. Learner posture recognition via a fusing model based on improved SILTP and LDP[J]. Multimedia Tools and Applications, 2019, 78(21):30443-30456.

7. Y. Cao, F. Xue, Y. Y. Chi, et al. Effective spatio-temporal semantic trajectory generation for similar pattern group identification[J]. International Journal of Machine Learning and Cybernetics, 2020, 11(2):287-300.

8. A. Partlow, C. Gibson, J. Kulon. 3D posture visualisation from body shape measurements using physics simulation, to ascertain the orientation of the pelvis and femurs in a seated position -ScienceDirect[J]. Computer Methods and Programs in Biomedicine, 2021, 198

9. Lin ChuanBi, Dong Ziqian, Kuan WeiKai, Huang YungFa. A Framework for Fall Detection Based on OpenPose Skeleton and LSTM/GRU Models[J]. Applied Sciences, 2020,11(1). 